\title{
GENETIC ALGORITHMS CAN BE USED TO OBTAIN GOOD LINEAR CONGRUENTIAL GENERATORS
}

\author{
J. C. Hernández, A. Ribagorda, P. Isasi*, and J. M. Sierra
}

ADDRESS: Computer Science Department, Carlos III University, Madrid SPAIN. \{jcesar, arturo, sierra\}oinf.uc3m.es *isasi@ia.uc3m.es.

ABSTRACT: Linear Congruential Generators (LCGs) are one model of pseudorandom number generators used in a great number of applications. They strongly depend on, and are completely characterized by, some critical parameters. The selection of good parameters to define a LCG is a difficult task mainly done, nowadays, by consulting tabulated values [10] or by trial and error.

In this work, the authors present a method based on genetic algorithms that can automatically solve the problem of finding good parameters for a LCG. They also show that the selection of an evaluation function for the generated solutions is critical to the problem and how a seemingly good function such as entropy could lead to poor results. Finally, other fitness functions are proposed and one of them is shown to produce very good results. Some other possibilities and variations that may produce fine linear congruential generators are also mentioned.

KEYWORDS: Pseudorandom number generator, linear congruential generator, genetic algorithms, fitness function, security, entropy, period, randomness, randomness testing.

\section{INTRODUCTION}

Since they were first proposed by Lehmer, in 1948 [11], Linear Congruential Generators (LCGs) are the most widely used Pseudorandom Number Generators (PRNGs). They are, also, one of the best analyzed models. Used in a great number of applications such as simulation, numerical analysis and optimization, they are frequently present where some PRNG is needed.

A typical Lineal Congruential Generator has the form:

$$
X_{n+1}=\left(a * X_{n}+b\right) \bmod m \text { with } X_{0}=\text { seed. }
$$

It is completely characterized by the parameters $\left(X_{0}, a, b, m\right)$. Actually, the value of $X_{0}$ is nearly irrelevant, as we will see when analyzing the MaxPeri- 
odTheorem that characterizes maximal LCGs, that is, LCGs that have maximal periods, i. e. periods of length $m$.

Although their cryptographic limitations $[1,7,16,17]$ are well known, for example, they are not recommended as key generators for stream ciphers, and behave poorly in some statistical tests, they remain in wide use, mainly because of their ease of implementation and very high speed. The best ones are, also, hard to find [15] because a badly chosen parameter usually makes the associated LCG worthless and, depending on the applications that will use it, even dangerous. In [9] one finds "Despite the large amount of theoretical research already done on this subject, many of the generators currently in use [...] are seriously flawed. Even some recently proposed or evaluated generators have a very weak theoretical justification." So selecting good parameters to define a LCG is quite a difficult task, and nowadays, if one wants to implement a LCG and knows something about the great importance of choosing good parameters, the only option available is to search through tabulated triples $(a, b, m)$ until finding one that suits one's needs. These tabulated triplets are chosen because of their good statistical properties when exposing the generated sequence of numbers to a battery of tests, but the tests vary too much in number and significance.

Lots of programmers found themselves tempted to choose these parameters at random, which is a very bad idea that implies that dozens of very poor LCGs around the world are doing their job badly. It seems that a new way of automatically finding good LCGs will be very useful.

The main idea of this work is to design a procedure for automatically finding good parameters $(a, b, m)$ for a LCG by using genetic algorithms, completely avoiding the use of tables. We also illustrate the feasibility of using genetic algorithms as a useful tool to find good parameters for certain algorithms, in this case a particular model of a pseudorandom number generator that is widely spread through thousands of applications around the world. We offer a new way to find different, but excellent and new LCGs. Also, more experienced users who want to use other LCGs that suits better their objectives than the tabulated one's can take advantage of this method.

\section{GENETIC ALGORITHMS}

Genetic Algorithms (GA) were first proposed by Holland [6] and since then they have been a subject of constant research and have been used in a wide range of applications [2, 14]. GA can be described as blind, stochastic and near-optimal heuristic search methods. GA do not work directly with the problem solutions, by the opposite, they use a codification of those solutions (usually in binary) 
called chromosomes. The searching process is based on a fitness function and the application of some operators called genetic operators. The fitness function is a numerical value that measures the success or fitness of a codified solution (a chromosome) to solve the problem. In these terms the GA works with sets of solutions called populations in which the individuals are step by step more accurate $[2,5]$, applying the genetic operators to successive populations. The evolution can be seeing, from a simplified perspective, like a process of genetic information transference between individuals [19]. This transference can be summarized in three rules:

1. selection of the more accurate individuals to be reproduced,

2. genetic recombination of the selected individuals and generation of new individuals containing this information, and

3. generation of new genetic information by altering randomly some old information in the chromosomes

GA use very little domain information (only the fitness function) about the problems to guide the searching process. This feature makes GA a good method for many different kinds of problems, specially in domains where there is little information about how to reach the goal, or where the domain could change on time. Some applications of GA have been proved more efficient when more information about the domain has been included. This information is often easily included by designing new ad hoc genetic operators for a specific problem. Even in those cases, the specific operators designed have showed to be useful in different domains.

\subsection{Mathematical description of the classical GA}

A simple, or canonical GA, could be described as a 7-tuple:

Where

$$
A G=\left(P^{0}, \lambda, I, s, \Omega, f, t\right) .
$$

$P^{0}=\left(a_{1}^{0}, \ldots, a_{\lambda}^{0}\right)$ is the initial population, $\lambda$ is the size of the population;

$I$ is the size of each individual's representation;

$s: P^{i} \rightarrow P^{j}$, where $P^{j} \subset P^{i}$, is the selection operator;

$\Omega=\left\{w_{1}, w_{2}, \ldots\right\}$ is the set of genetic operators that create new individuals (for example crossover and mutation), and it holds that for all $k, w_{k}: P^{i} \rightarrow$ $P^{j}$ and there exists $a_{m}^{j}$ such as $a_{m}^{j} \in P^{j}$ and $a_{m}^{j} \notin P^{i}$;

$f: a_{i}^{j} \rightarrow \Re$ is the fitness function. 
$t: P^{i} \rightarrow\{$ Yes, No $\}$ is the convergence criteria that decides whether to continue or stop the algorithm execution. Usually the criterion for stopping is to reach a given number of generations or some measure of the similarity between the individuals of one population.

\subsection{Genetic operators}

There are three main genetic operators used to guide the searching procedure:

\subsubsection{Selection}

It is an implementation of Darwin's Natural Selection of the fittest. It consists of selecting the best individuals in the population to generate an intermediate population named matting pool. The matting pool is of the same size as the population and is generated selecting individuals from the population at random but proportionally to their fitness value. In this way there would be more copies, in the matting pool, of better individuals in terms of fitness.

\subsubsection{Crossover}

Individuals in the matting pool are coupled crossed-over to generate new individuals that share some genetic material from one parent and some from the other. These new individuals are included in the new generated population.

In the following example individuals $i_{1}$ and $i_{2}$ are crossed-over in alele number 5 to generate two new individuals $n i_{1}$ and $n i_{2}$ that are included in the new population:

$$
\begin{aligned}
& i_{1} \rightarrow\{11101\} 001000 \\
& i_{2} \rightarrow 00001\{010101\}
\end{aligned}
$$

generate

$n i_{1} \rightarrow 11101010101$ (first 5 bits of $i_{1}$ with last 6 bits of $i_{2}$ )

$n i_{2} \rightarrow 00001001000$ (first 5 bits of $i_{2}$ with last 6 bits of $i_{1}$ ).

\subsubsection{Mutation}

This operator randomly changes the value of a bit of an individual. The utility of this operator is to increase the genetic diversity of populations, avoiding the biases that could exist in the initial population. Another important effect is to make the searching process more exploratory. There exists a mutation probability that is applied to all the bits in the population. The higher the probability, the 
more exploratory the process will become. The mutation probability has usually some low fixed value, but some works, notably [12], have proved the advantage of an adaptive mutation probability.

\subsection{GA general working procedure}

A generation in a GA can be described as follows:

1. A population of $\mathrm{N}$ individuals is generated randomly in generation number 0 .

2. A matting pool of $\mathrm{N}$ individuals is generated, selecting individuals from the population by means of the selection genetic operator.

3. Two individuals from the matting pool are selected at_random and removed from the matting pool. These two individuals are then crossed-over and the two new offspring generated included in a new population.

If the matting pool is not empty go to (3).

4. Apply the mutation operator to all the elements of the new population.

5. Substitute the old population with the new generated in (3-5).

6 . If the convergence criteria has not been reached yet go to (2), otherwise stop.

\section{FITNESS FUNCTIONS FOR OUR PROBLEM.}

Genetic algorithm-based methods are a natural approach when one must find the best (or at least a very good) solution to a problem between a really wide number of possibilities. One can also think of genetic algorithms as a way of doing a guided search through the space of tridimensional vectors $(a, b, m)$ in natural numbers that, if well designed, is much better than exhaustive searching.

The main difficulty when working with a genetic algorithm is usually the finding of an adequate fitness function, that is, the function that will sort different individuals with respect to their probabilities of being close to the best solution. There are also other problems to solve like the representation problem (Which is the best representation of the data and solutions?), and the tuning of the genetic algorithm's own parameters (for example, which mutation probability will produce better results). 


\subsection{Selecting LCGs}

In our particular case we have an additional problem, because good or bad LCGs are sometimes difficult to distinguish and, frequently, this classification depends highly on the application that the LCG is going to have. Good LCGs for a certain application can be considered bad for others and vice-versa. For example, many commonly used LCGs (including DRAND48, available in some versions of the UNIX operating system) use a modulus that is a power of two because this makes the LCG fast and convenient to implement in a computer. However, this produces highly correlated low order bits because the last bit simply switches from 0 to 1 . This can cause problems in certain types of simulations or in applications that depend on the randomness of these low-order bits, but could be adequate for applications that require float pseudorandom numbers for which the low-order bits are completely irrelevant. So, for applications that rely on the randomness of the low order bits even good LCGs can behave poorly. Anyway, these problems can be easily avoided by selecting a prime modulus.

The ideal fitness function would be one capable of perfectly measuring the randomness of a LCG in question. Unfortunately this function, one universal randomness-measuring function, simply does not exist. One cannot measure randomness directly, in a single variable. Randomness is a hard concept which is, also, highly multidimensional. We must try different approximations.

For a given pseudorandom number generator, be it a LCG or whatever, the usual rule of thumb is to make it pass a given battery of tests and see the results. These tests reject most of the generators as non-random, but offer no guarantees on the generators that pass the tests. One generator (or in our case, a triplet of parameters $a, b$ and $m$ ) that passes one hundred tests and performs as being random can behave very poorly in the next test and be rejected with strong evidence as being non-random. This battery testing method is the only way of accepting or rejecting generators, but it is clearly not good.

\subsubsection{Statistical tests}

In order to have a measure of the goodness of a given LCG, the following statistical tests have been used:

1. Entropy: The information density of the output of one cycle of the generator, expressed as the number of bits by byte (pack of 8 bits) generated, so it can take any value between 0.0 and 8.0 , being higher values better but exponentially harder to reach by chance. It is one widely used way to measure one aspect of randomness. Mathematically, it is expressed by the formula $\sum_{x \in G F\left(2^{8}\right)}-p(x) \log _{2} p(x)$. 
2. Chi-Square percentile: The Chi-Square test is a very commonly used test for the randomness of data because it is extremely sensitive to errors in pseudorandom number generators. Its statistic compares the observed probabilities of every byte in a cycle of the generator against the expected ones if it were uniformly distributed. The test evaluates the probability that the observed value of the statistic will be obtained from a uniform distribution. Values higher than $99 \%$ or less than $1 \%$ indicate that the sequence is almost certainly not random, and values between $99 \%$ and $95 \%$ or between $5 \%$ and $1 \%$ suggest that the sequence is suspect. The ideal value is $50 \%$ but values obtained in the $25 \%-75 \%$ interval can be considered good enough to pass the test. No LCG pass this test.

3. Arithmetic mean: Simply the arithmetic mean of all the values in a cycle of a generator. As every value between 0 and 255 must be equiprobable, results close to $255 / 2=127.5$ must be obtained if the generator has good properties.

4. Monte Carlo value for $\pi$ : This test simply takes each successive sequence of six bytes and transform it into two 24 bits coordinates $(X, Y)$ that generate a point within a square. If the distance of this pseudorandomly generated point is less than the radius of the circle inscribed whit in the square, this point is considered a hit. Repeating this process a large number of times, the percentage of hits can be used to calculate the value of $\pi$, and if the sequence is close to random this calculation will slowly converge to the real value of $\pi$.

5. Serial correlation coefficient: This quantity measures the extent to which each byte in the file depends upon the previous byte. For random sequences, this value (being it positive or negative) must be close to zero.

6. Maximal Period: A LCG determined by parameters $a, b$ and $m$ has maximal period if and only if the length of its period, that is, the length of the sequence that it produces before repeating, is $m$. To do this test we examine the sequence, looking for sub-cycles or degenerate periods or some mathematical properties, to determine its period. If we found a maximum period we labeled this column with a ' $\mathrm{Y}$ ', otherwise with a ' $\mathrm{N}$ '.

7. Percentage of the maximum period achieved: A little redundant with test 6 , but useful if the generator does not have a maximal period.

\subsection{Entropy as a fitness function}

We propose to slightly modify Shannon's classical definition of entropy to be, in our case, the information density of the output of one cycle of a given generator, 
expressed mathematically by the formula:

$$
\sum_{x \in G F(2)}-p(x) \log _{2} p(x)
$$

where $p(x)$ is the observed probability of $x$ in one cycle of the LCG.

We thought that entropy would be a very good fitness function. A LCG can be seen as a source of randomness and, obviously, higher entropy values are better. One thing worth mentioning is how entropy is measured. If we measure per bit entropy (as in the formula above) our maximum value will be 1.0 and will be easily reached by all sequences with the same number of 0 's and 1's. This seems not to be a good choice as something like $10101010101010101010 \ldots . .10$ will have maximum entropy, being obviously quite predictable and, hence, non-random. So it is important to measure entropy at higher, more difficult, levels. We found that per byte entropy (measuring the entropy of consecutive blocks of 8 bits) was quite adequate, since reaching the maximum of 8.0 was exponentially more difficult and much more informative. So our new formula is

$$
\sum_{x \in G F\left(2^{8}\right)}-p(x) \log _{2} p(x) .
$$

We also limited the range of integers to study because we defined our chromosome length to be 45 bits long. These 45 bits were logically divided into three groups to include the binary representation of $a, b$, and $m$ (in this order) so this bounded each $a, b$, and $m$ to be less than or equal to $2^{15}-1$. This has no effect on our results except for the fact that better LCGs are achievable when larger integers can be used: larger periods can only be obtained if we can choose between larger $m$ 's and better or equal multipliers can be found if we have a higher upper bound. So we must compare our results against LCGs of our size. Anyway, our results are easily generalized to larger values just by extending the chromosome length.

Using this entropy-based fitness function we started to run our genetic algorithm implementation with what we observed to be good internal parameters, found after a number of trials and errors that pointed out that a crossover probability of 1.0 (100\%), a low mutation probability (around 0.05 or a $5 \%$ ), and a population size of 50 individuals were a good choice (because it tends to produce the best solutions). We also used some elitism (the two best individuals always passed directly to the next generation) and tournament selection. The finishing criterion was the reaching of 10.000 generations because we observed that after that limit the populations did not significantly evolve and no better solutions were found. 
We obtained surprisingly good results, that is, results that clearly surpassed the best of our expectations. In Table $1 \mathrm{~A}$ and Table $1 \mathrm{~B}$ below we see the results of the 5 statistical tests we performed on a series of recommended LCGs that we got from tables in [18], and in Tables $2 \mathrm{~A}$ and Table $2 \mathrm{~B}$ we see the results of the same tests on the LCGs we obtained with this entropy only based approach.

At first sight the results on Table $2 \mathrm{~A}$ are much better (see explanations in section 3.1.1) than the results on Table 1A: all Chi-square percentiles in Table 2A are equal or better than those in Table 1A, the worst values for the entropy, arithmetic mean, and Monte Carlo $\pi$ estimation on Table 2A are much better than the best in Table 1A, etc. So one is tempted to conclude research at this point pretending to have much better LCGs than the tabulated (and recommended) ones.

\begin{tabular}{|c|c|c|c|c|c|c|}
\hline $\mathrm{a}$ & $\mathrm{b}$ & $\mathrm{m}$ & 1 & 2 & 3 & 4 \\
\hline \hline 106 & 1283 & 6075 & 7.955 & 0.01 & 133.8 & $4.29 \%$ \\
\hline 1366 & 1283 & 6075 & 7.957 & 0.01 & 133.1 & $2.56 \%$ \\
\hline 936 & 1399 & 6655 & 7.961 & 0.01 & 133.5 & $3.43 \%$ \\
\hline 211 & 1663 & 7875 & 7.954 & 0.01 & 136.5 & $6.94 \%$ \\
\hline 421 & 1663 & 7875 & 7.954 & 0.01 & 136.0 & $7.20 \%$ \\
\hline 430 & 2531 & 11979 & 7.972 & 0.01 & 133.5 & $3.42 \%$ \\
\hline 859 & 2531 & 11979 & 7.973 & 0.01 & 133.6 & $4.14 \%$ \\
\hline 1741 & 2731 & 12960 & 7.977 & 0.01 & 132.2 & $2.08 \%$ \\
\hline 1541 & 2957 & 14000 & 7.973 & 0.01 & 133.6 & $3.33 \%$ \\
\hline \hline 967 & 3041 & 14406 & 7.971 & 0.01 & 134.2 & $4.24 \%$ \\
\hline \hline 1291 & 4621 & 21870 & 7.983 & 0.01 & 131.8 & $2.48 \%$ \\
\hline \hline 419 & 6173 & 29282 & 7.979 & 0.01 & 133.6 & $2.81 \%$ \\
\hline \hline 1255 & 6173 & 29282 & 7.979 & 0.01 & 133.9 & $4.93 \%$ \\
\hline \hline 625 & 6571 & 31104 & 7.975 & 0.01 & 134.4 & $4.55 \%$ \\
\hline
\end{tabular}

Table 1A:

Test 1: Per byte entropy (optimum $=8.0$ ).

Test 2: Chi-square percentile.

Test 3: Arithmetic mean (optimum $=127.5$ ).

Test 4: Error (\%) in the estimation of $\pi$ by a Monte Carlo method. 


\begin{tabular}{|c|c|c|c|}
\hline $\mathrm{a}$ & $\mathrm{b}$ & $\mathrm{m}$ & 5 \\
\hline \hline 106 & 1283 & 6075 & -0.0127 \\
\hline 1366 & 1283 & 6075 & 0.0026 \\
\hline 936 & 1399 & 6655 & -0.0036 \\
\hline 211 & 1663 & 7875 & -0.0059 \\
\hline 421 & 1663 & 7875 & -0.0199 \\
\hline 430 & 2531 & 11979 & 0.0010 \\
\hline 859 & 2531 & 11979 & 0.0061 \\
\hline 1741 & 2731 & 12960 & -0.0081 \\
\hline 1541 & 2957 & 14000 & -0.0154 \\
\hline \hline 967 & 3041 & 14406 & -0.0078 \\
\hline \hline 1291 & 4621 & 21870 & -0.0043 \\
\hline \hline 419 & 6173 & 29282 & -0.0052 \\
\hline \hline 1255 & 6173 & 29282 & -0.0106 \\
\hline \hline 625 & 6571 & 31104 & -0.0133 \\
\hline
\end{tabular}

Table 1B:

Test 5: Serial Correlation Coefficient (optimum $=0.0$ ).

\begin{tabular}{|c|c|c|c|c|c|c|}
\hline $\mathrm{a}$ & $\mathrm{b}$ & $\mathrm{m}$ & 1 & 2 & 3 & 4 \\
\hline \hline 7476 & 3206 & 21497 & 7.991 & 0.01 & 129.5 & $1.79 \%$ \\
\hline 6943 & 5593 & 22873 & 7.990 & 0.01 & 128.0 & $1.06 \%$ \\
\hline 15131 & 6914 & 22873 & 7.993 & 0.01 & 128.6 & $1.05 \%$ \\
\hline 7476 & 2019 & 21331 & 7.992 & 0.01 & 129.7 & $0.96 \%$ \\
\hline 26977 & 10516 & 20817 & 7.993 & 2.50 & 129.3 & $0.46 \%$ \\
\hline 23989 & 1911 & 21331 & 7.993 & 0.50 & 129.1 & $0.91 \%$ \\
\hline 14684 & 29655 & 29241 & 7.993 & 0.01 & 127.5 & $0.87 \%$ \\
\hline
\end{tabular}

Table 2A:

Test 1: Per byte entropy (optimum $=8.0$ ).

Test 2: Chi-square percentile.

Test 3: Arithmetic mean (optimum = 127.5).

Test 4: Error (\%)in the estimation of $\pi$ by a Monte Carlo method 


\begin{tabular}{|c|c|c|c|c|c|}
\hline $\mathrm{a}$ & $\mathrm{b}$ & $\mathrm{m}$ & 5 & 6 & 7 \\
\hline \hline 7476 & 3206 & 21497 & -0.0163 & $\mathrm{~N}$ & $6.80 \%$ \\
\hline 6943 & 5593 & 22873 & 0.0664 & $\mathrm{~N}$ & $3.11 \%$ \\
\hline 15131 & 6914 & 22873 & 0.0119 & $\mathrm{~N}$ & $6.23 \%$ \\
\hline 7476 & 2019 & 21331 & -0.0210 & $\mathrm{~N}$ & $12.30 \%$ \\
\hline 26977 & 10516 & 20817 & -0.0227 & $\mathrm{~N}$ & $6.23 \%$ \\
\hline 23989 & 1911 & 21331 & 0.0014 & $\mathrm{~N}$ & $12.30 \%$ \\
\hline 14684 & 29655 & 29241 & -0.0512 & $\mathrm{~N}$ & $3.51 \%$ \\
\hline
\end{tabular}

Table 2B:

Test 5: Serial Correlation Coefficient (optimum $=0.0$ ).

Test 6: Does it have maximal period? Yes or No.

Test 7: $\%$ of the maximal period achieved (optimum $=100 \%$ ).

This is not true. These generators look quite impressive, but they are very bad indeed. In spite of its well-known use in coding theory and cryptography, and against first intuition, entropy is not a definite randomness measuring function. It is obvious that, in our case, generators that produce very low entropy values must be rejected as non-random (because they produce non uniform output when they should), but, as it happens with any other test, high values of entropy do not prove randomness at all. In fact, entropy measures are nothing more than another randomness test, not better nor worse than any other.

In our case, not only entropy values are significantly better on Table $2 \mathrm{~A}$, as one can expect when using genetic algorithms to maximize entropy, also most of the rest. The worst (farthest from ideal values that would have been obtained from random sources) value on Table 2A for tests 1,2,3 and 4 is much better (or at least equal) than the best one in Table $1 \mathrm{~A}$. The only significant exception is test 5 (serial correlation coefficient), but apparently, correlation values in Table $2 \mathrm{~B}$ are quite good too (very near to zero). It seems as if there is strong evidence in favor of Table 2A generators, but in fact they are very poor. They do not have maximal periods (as shown on Table $2 \mathrm{~B}$ in tests 6 and 7 ). The values of the serial correlation coefficient reflect this undesirable behavior.

Not having maximal period is not really so bad, if the period is quite close to this maximum. But this is not the case. A look at Table 2B shows these generators are not good because none of them achieve even $13 \%$ of its theoretical maximum period. We must reject all these generators as being not useful for any uses (except, perhaps, exemplification of the limited value of entropy measures and, by extension, randomness tests ).

But how can we know a priori if a given LCG will have a maximum period? The characterization of a maximal period LCG is done by the next Max Period 
Theorem.

MaxPeriodTheorem: (Taken from [8])

The linear congruential sequence

$$
X_{n+1}=\left(a * X_{n}+b\right) \bmod m \text { with } X_{0}=\text { seed }
$$

defined by $m, a, b$ and $X_{0}$ has maximal period (i.e. period length $m$ ) if and only if:

i) $b$ is relatively prime to $m$,

i) $a-1$ is a multiple of $p$, for every prime $p$ dividing $m$, and

iii) $a-1$ is a multiple of 4 if $m$ is a multiple of 4 .

\subsection{New fitness functions}

Using an entropy-only based fitness function we do not get maximal period generators, contrary to our first beliefs that entropy is a definite randomness measure. So if we want maximal period generators we must ask for it in the fitness function. We must obviously change our fitness function to include something related to the period of the LCG. One of our first attempts was the use of

$$
\text { fitness }=\text { entropy }+ \text { period, }
$$

but this function was quickly rejected because our search degenerated soon to nearly maximal $m$ 's (in our implementation, the maximum value for $m$ is $2^{15}-1=$ 32767 ). This was due to the very low significance that $0.0 \leq$ entropy $\leq 8.0$ had in this fitness function where $0 \leq$ period $\leq 32767$ become much more important (32767 parts of 32775 , a weighted value of about $99.976 \%$ ) so there is a very strong selective pressure for the GA towards maximizing only the period. As expected, the GAs with this fitness function generated LCGs with long period but low entropy, typically about the value of $7.4+32760$.

A better idea is to use a normalized measure of the period. Then, our fitness could be

$$
\text { fitness }=\text { entropy }+\frac{\text { period }}{\text { maxperiod }}=\text { entropy }+\frac{\text { period }}{m} \text {. }
$$

(as $m$ is the maximum period achievable by our LCG) and will have a maximum of 9.0. We tried this fitness function and found that, although it produced interesting results, it suffered from nearly the same problems as our first only entropy-dependent function, as it gave much more importance to entropy (8 
parts of 9) than to period ( 1 parts of 9) and we felt both measures were equally important.

The next and final fitness function we used was, then,

$$
\text { fitness }=\text { entropy } \cdot \frac{\text { period }}{\operatorname{maxperiod}}=\text { entropy } \cdot \frac{\text { period }}{m} \text {. }
$$

This balances nicely both measures. Now our new maximum was 8.0 (but it was more difficult to obtain at random because

$$
0 \leq \frac{\text { period }}{\text { maxperiod }} \leq 1
$$

\section{RESULTS}

When using this final fitness function proposed above, we got lots of interesting generators, some of which are shown in Table 3A below:

\begin{tabular}{|c|c|c|c|c|c|c|}
\hline $\mathrm{a}$ & $\mathrm{b}$ & $\mathrm{m}$ & 1 & 2 & 3 & 4 \\
\hline \hline 15005 & 8371 & 19993 & 7.983 & 0.01 & 132.7 & $4.26 \%$ \\
\hline 6237 & 10697 & 21023 & 7.985 & 0.01 & 132.9 & $5.12 \%$ \\
\hline 14359 & 9654 & 21569 & 7.985 & 0.01 & 132.3 & $3.49 \%$ \\
\hline 12586 & 11658 & 21023 & 7.985 & 0.01 & 132.7 & $5.25 \%$ \\
\hline 4518 & 15578 & 21179 & 7.985 & 0.01 & 132.0 & $2.30 \%$ \\
\hline 1271 & 10331 & 20983 & 7.985 & 0.01 & 132.3 & $3.69 \%$ \\
\hline 6533 & 4712 & 21011 & 7.985 & 0.01 & 132.9 & $4.32 \%$ \\
\hline 14945 & 6262 & 21089 & 7.985 & 0.01 & 132.5 & $2.10 \%$ \\
\hline 1 & 19568 & 19647 & 7.984 & 0.01 & 132.8 & $3.47 \%$ \\
\hline
\end{tabular}

Table 3A:

Test 1: Fitness (optimum = 8).

Test 2: Chi-square percentile.

Test 3: Arithmetic mean (optimum $=127.5$ ).

Test 4: Error (\%) in the estimation of $\pi$ by a Monte Carlo method. 


\begin{tabular}{|c|c|c|c|c|c|}
\hline $\mathrm{a}$ & $\mathrm{b}$ & $\mathrm{m}$ & 5 & 6 & 7 \\
\hline \hline 15005 & 8371 & 19993 & -0.0007 & $\mathrm{Y}$ & $100.00 \%$ \\
\hline 6237 & 10697 & 21023 & -0.0017 & $\mathrm{Y}$ & $100.00 \%$ \\
\hline 14359 & 9654 & 21569 & -0.0024 & $\mathrm{Y}$ & $100.00 \%$ \\
\hline 12586 & 11658 & 21023 & 0.0025 & $\mathrm{Y}$ & $100.00 \%$ \\
\hline 4518 & 15578 & 21179 & -0.0005 & $\mathrm{Y}$ & $100.00 \%$ \\
\hline 1271 & 10331 & 20983 & -0.0022 & $\mathrm{Y}$ & $100.00 \%$ \\
\hline 6533 & 4712 & 21011 & -0.0103 & $\mathrm{Y}$ & $100.00 \%$ \\
\hline 14945 & 6262 & 21089 & -0.0089 & $\mathrm{Y}$ & $100.00 \%$ \\
\hline 1 & 19568 & 19647 & 0.0052 & $\mathrm{Y}$ & $100.00 \%$ \\
\hline
\end{tabular}

Table 3B:

Test 5: Serial Correlation Coefficient (optimum $=0$ ).

Test 6: Does it have maximal period? $Y / N$.

Test 7: $\%$ of the maximal period achieved (optimum $=100 \%$ ).

The results of the tests we performed on these generators are better but less spectacular than those of Table 2A. This is exactly what we were looking for: if we compare our generators of Table 3A against those shown (and recommended in the literature as good generators) on Table $1 \mathrm{~A}$ we see they perform very close, our's slightly better in all the tests. They even pass the difficult serial correlation test all the generators of Table 2B clearly failed. Our aim was to provide an automatic method of generating LCGs as good as the tabulated ones and the results on Table $3 \mathrm{~A}$ and $3 \mathrm{~B}$ prove that, at least from these tests' point of view, we have succeed.

\subsection{Two interesting facts}

We believe it is worthy to mention two curious facts: All the $m$ 's, except the last, obtained by our entropy+period approach and shown on Table 3A and Table 3B have one interesting property in common: they are all prime numbers. That is a very direct and clever way of assuring that all the requisites in MaxPeriodTheorem are satisfied. Our genetic algorithm has found an intelligent and direct approach to meet all these requisites. Genetic algorithms have shown extremely high power in finding extraordinary bad LCGs with incredible good entropy, that is, finding exceptions. They also usually prefer the shortest, quickest solutions. In our case, during crossover, due to the representation chosen, around two in three times the $m$ of one of the parents is passed to the child. Being $m$ so important as to assure maximal period (if $m$ prime and $b>0$ ), good $m$ 's are quickly spread through the population as a very good characteristic. When two individuals with the same (prime) $m$ have crossover applied, the same $m$ will be 
inherited by the child, thus guaranteeing a good average fitness, and only $a$ or $b$ are changed, thus searching for optimal $a$ 's and b's for a given $m$.

Obviously, $m$ is not forced to be prime by the genetic algorithm. It can take any other value, producing different generators, but prime $m$ 's are more likely. This is simply a shortcut that genetic algorithms have found to easily achieve MaxPeriodTheorem requisites.

In our last example we have a non-prime $m$, but in this case maximum period is achieved by selecting $a=1$, thus assuring all those requisites on $a-1$ (in this case 0 , so multiple of any number) of the MaxPeriodTheorem in a very nice and direct way.

\section{FURTHER RESEARCH}

Although this is a preliminary study in this subject, we feel it is interesting as a proof of concept. Some changes could be made that may improve the results obtained without changing the main ideas behind this research. We will mention. here two of these changes that will probably lead to further and interesting research:

- Increasing the size of the chromosomes will obviously produce better or equal LCGs, as explained before. This can be accomplished easily, and the results we would obtain could probably be comparable with the best LCGs known.

- Changing the fitness function to reflect a preference for greater values of $m$ could be very interesting or even necessary in some applications. It also can be done easily, say adding a term like $\frac{1}{15} * \log (m)$ to the fitness function we have proposed.

The authors strongly believe that variants of this method can be easily developed to help in the parameter choosing of other types of generators like Lagged Fibonacci Generators [13], ICGs [3] or EIGCs [4]. All these ideas will be explored in the future.

\section{CONCLUSIONS}

We believe we have provided strong evidence that passing a series of tests, call them poker test, chi-square or even entropy, does not assure the passing of further tests and that excellent results on some particular tests do not necessarily mean anything about the randomness of a generator. 
We also have shown that a method relatively new in this field, the use of genetic algorithms, can be of great help when looking for good parameters. After the work of Pierre L'Ecuyer [9] in combining LCGs to improve their properties, this automatic way of getting good and little LCGs drastically increases its interest, as it could be used to automatically generate a pool of good LCGs that then can be combined to make an even better generator.

\section{REFERENCES}

1. Boyar, J. 1989. Inferring sequences produced by pseudorandom number generators. J. Assoc. Comput. Mach.. 36(1): 129-141.

2. Chambers, L. D. (ed.) 1995. Practical Handbook of Genetic Algorithms. Boca Raton FL: CRC. Press.

3. Eichenauer-Herrman, J. 1991. Inversive congruential pseudorandom numbers avoid the planes. Math. Comp. 56: 297-301.

4. Eichenauer-Herrman, J. 1993. Explicit inversive congruential pseudorandom numbers: The compound approach. Computing. 51: 175-182.

5. Goldberg, D. E. 1989. Genetic Algorithms in search, optimization and machine learning. Reading MA: Addison-Wesley.

6. Holland, J. H. 1975. Adaptation in Natural and Artificial Systems. Ann Arbor MI: The University of Michigan Press.

7. Knuth, D. E. 1980. Deciphering a Linear Congruential Encryption. Technical Report 024800. Stanford CA: Stanford University.

8. Knuth, D. E. 1981. The Art of Computer Programming. Second Edition.. Reading PA: Addison-Wesley. p. 16

9. L'Ecuyer, P. 1988. Efficient and Portable Combined Random Number Generators. Communications of the ACM. 31: 742-749.

10. L'Ecuyer, P. 1999. Tables of Linear Congruential Generators of Different Size and Good Lattice Structure. Mathematics of Computation. 68: 249-260.

11. Lehmer, D. H. 1951. Mathematical methods in large-scale computing units. In Proc. 2nd. Sympos. on Large-Scale Digital Calculating Machinery Cambridge MA 1949. Cambridge MA: Harvard University Press. pp. 141-146.

12. Maley, C. 1995. The coevolution of mutation rates. In Advances in Artificial Life. New York NY: Springer-Verlag. pp. 234-245.

13. Mascagni, et al. 1995. A fast, high-quality and reproducible Lagged Fibonacci pseudorandom number generator. J. Comput. Physics. 15: 211-219.

14. Mitchell, M. 1996. An introduction to Genetic Algorithms. Cambridge MA: MIT Press. 
15. Park and Miller. 1988. Random Number Generators: Good Ones Are Hard to Find. Communications of the ACM. 31(10): 1192-1201.

16. Plumstead, J. B. 1983. Inferring a Sequence Generated by a Linear Congruence. In David Chaum, Ronald Rivest, and Alan T. Sherman (Editors) Proceedings of the 23rd IEEE Symposium on the Foundations of Computer Science, August 1982. Plenum Press: New York. pp. 317-319.

17. Reeds, J. 1977. Cracking a Random Number Generator. Cryptologia. 1: $20-26$.

18. Scheirer, B. 1996. Applied Cryptography. 2nd Edition. New York NY: John Wiley \& Sons. p. 348

19. Wilson, S. W. 1985. Knowledge growth in an artificial animal. In J. Grefenstette (Ed.) Proceedings of the First International Conference on Genetic Algorithms and Their Applications, July 1985. Hillsdale NJ: Lawrence Erlbaum Associates.

\section{BIOGRAPHICAL SKETCHES}

Julio César Hernández Castro is a $\mathrm{PhD}$ student at, and a member of, the Security Group of the Computer Science Department of Carlos III University at Madrid SPAIN. He is mainly interested in Cryptography and on the potential applications of artificial intelligence techniques to cryptology and cryptanalysis.

Dr. Arturo Ribagorda Garnacho is a Professor at the Security Group of the Computer Science Department of Carlos III University at Madrid, Spain. He has published four books about computer security and cryptography and more than 30 articles in international workshops and congresses. His research interests are network security and cryptography.

Dr. Pedro Isasi Viñuela is a Professor at the Artificial Intelligence Group of the Computer Science Department of Carlos III University at Madrid SPAIN. He is specialized in evolutionary computation and, in particular, in genetic algorithms. He has more than 30 international publications, mainly in the use of genetic algorithms to real-world applications.

Dr. José María Sierra Cámara is a Lecturer at the Security Group of the Computer Science Department of Carlos III University. His current research interests are cryptography and network security, specially the IPv6 and IPSEC protocols. 